\title{
Ectopic expression of an EAR motif deletion mutant of SIERF3 enhances tolerance to salt stress and Ralstonia solanacearum in tomato
}

\author{
I-Chun Pan · Chia-Wen Li • Ruey-Chih Su • \\ Chiu-Ping Cheng • Choun-Sea Lin • \\ Ming-Tsair Chan
}

Received: 17 June 2010/ Accepted: 16 July 2010

(C) Springer-Verlag 2010

\begin{abstract}
Ethylene-responsive transcription factors (ERFs) bind specifically to cis-acting DNA regulatory elements such as GCC boxes and play an important role in the regulation of defense- and stress-related genes in plants. In contrast to other ERFs, class II ERFs contain an ERF-associated amphiphilic repression (EAR) domain and act as GCC-mediated transcriptional repressors. In this study,
\end{abstract}

I.-C. Pan and C.-W. Li contributed equally to this work.

Electronic supplementary material The online version of this article (doi:10.1007/s00425-010-1235-5) contains supplementary material, which is available to authorized users.

\section{I.-C. Pan · M.-T. Chan}

Institute of Biotechnology, National Cheng Kung University,

Tainan 70101, Taiwan

I.-C. Pan · M.-T. Chan

Agricultural Biotechnology Research Center,

Academia Sinica, Taipei 11529, Taiwan

I.-C. Pan · C.-W. Li · M.-T. Chan ( $\square)$

Academia Sinica Biotechnology Center in Southern Taiwan,

Tainan 74146, Taiwan

e-mail: mbmtchan@gate.sinica.edu.tw

R.-C. Su

Department of Life Science, Fu Jen Catholic University,

Taipei 24205, Taiwan

C.-P. Cheng

Department of Life Science, Graduate Institute of Plant Biology,

National Taiwan University, Taipei 10617, Taiwan

C.-S. Lin

Agricultural Biotechnology Research Center,

Academia Sinica, Taipei 11529, Taiwan
SlERF3, a class II ERF was isolated from tomato and characterized. To examine whether the EAR motif of class II ERF proteins participates in ERF-mediated functions in plants, the EAR domain was deleted to generate SIERF3 $\triangle R D$. We show that SIERF3 $\triangle R D$ protein retains the character of a transcription factor and becomes a GCC-mediated transcriptional activator. Constitutive expression of full-length SIERF3 in tomato severely suppressed growth and, as a result, no transgenic plants were obtained. However, no apparent effects on growth and development of SlERF3 $\triangle R D$ transgenic plants were observed. Overexpression of SIERF3 $\triangle R D$ in transgenic tomato induced expression of pathogenesis-related protein genes such as $P R 1, P R 2$ and $P R 5$, and enhanced tolerance to Ralstonia solanacearum. Furthermore, transgenic Arabidopsis and tomatoes constitutively expressing SlERF3 $\triangle R D$ exhibited reduced levels of membrane lipid peroxidation and enhanced tolerance to salt stress. In comparison with wild-type plants grown under stress conditions, transgenic SlERF3 $\triangle R D$ tomatoes produced more flowers, fruits, and seeds. This study illustrates a gene-enhancing tolerance to both biotic and abiotic stresses in transgenic plants with the deletion of a repressor domain. Our findings suggest that class II ERF proteins may find important use in crop improvement or genetic engineering to increase stress tolerance in plants.

Keywords AP2/ERF - EAR - Pathogen resistance . Repression domain $\cdot$ Salt tolerance
Abbreviations
ERF Ethylene-responsive factor
EAR ERF-associated amphiphilic repression
JA Jasmonic acid
SA Salicylic acid 


\section{Introduction}

The ethylene-responsive factor (ERF) family, a large transcription factor gene family, belongs to the AP2/ERF superfamily, which is defined by the highly conserved AP2 DNA-binding domain consisting of 60-70 amino acid residues (Jofuku et al. 1994; Sakuma et al. 2002). According to the number of AP2/ERF domains, the AP2/ERF superfamily is divided into ERF, AP2, and RAV families (Sakuma et al. 2002; Nakano et al. 2006). The ERF family is further classified into two subfamilies: dehydration-responsive elementbinding protein (DREB) and ERF subfamilies. The former is involved in hormonal signal transduction and plant responses to abiotic stresses (Hsieh et al. 2002b; Narusaka et al. 2003; Qin et al. 2008), and the latter is involved in both plant defense- and stress-signaling pathways (Yang et al. 2005; Onate-Sanchez et al. 2007; Pre et al. 2008).

Previous studies have reported that members of the AP2/ ERF superfamily involved in the transcription of downstream genes via binding to cis-acting promoter elements such as GCC, CRT/DRE, JERE, or VWRE (Ohme-Takagi and Shinshi 1995; van der Fits and Memelink 2001; Gu et al. 2002; Sasaki et al. 2007). Based on the amino acid sequence analysis, Fujimoto et al. (2000) and Tournier et al. (2003) categorized ERF proteins into four classes. Among them, class II ethylene-responsive transcription factors (ERFs) contain a conserved repressor domain, L/FDLNL/ $\mathrm{F}(\mathrm{x}) \mathrm{P}$, termed ERF-associated amphiphilic repression (EAR) motif or CMVIII-1 motif, at the $\mathrm{C}$ terminus. This group of ERF proteins containing the EAR motif was later classified as B1-1a group (Nakano et al. 2006).

In contrast to other ERFs acting as transcriptional activators, EAR-containing ERFs act as a GCC-mediated transcriptional repressor (Fujimoto et al. 2000; Ohta et al. 2001). Several class II ERFs have been isolated and proved to be transcriptional repressors such as AtERF4, AtERF7, AtERF10, AtERF11, AtERF12, and NtERF3 (Ohta et al. 2001; McGrath et al. 2005). Furthermore, the fusion of different activation domains of various transcription factors with EAR could also repress the transcription of specific target genes (Ohta et al. 2001; Yang et al. 2005), and even result in loss-of-function phenotypes in transgenic plants (Hiratsu et al. 2003). Recently, the EAR motif has been found to convert a transcriptional complex into a transrepressor (Matsui and Ohme-Takagi 2009).

Similar to other AP2/ERF transcription factors, EARcontaining ERFs can play an important role in the regulation of defense- and stress-related genes in plants. For instance, SodERF3 can be induced by ABA, salt, and wounding. Constitutive expression of sugarcane SodERF3 increased tolerance to drought and osmotic stress in transgenic tobacco (Trujillo et al. 2008). The transcripts of cotton GhERF4 gene are rapidly increased after salt, ethylene, cold, drought, and ABA treatment (Jin and Liu 2008). The expression of rice OsBIERF4 genes is induced by salicylic acid (SA) and by Magnaporthe grisea infection (Cao et al. 2006). Additionally, RNA expression of $L e E R F 3 b$ is regulated by fruit ripening and environmental stresses (Chen et al. 2008). The function of EAR-containing genes in response to biotic and abiotic stresses remains to be individually clarified. Recently, repressors have been considered to function as safety controllers that prevent damage from activation of programmed cell death caused by runaway response pathways in plants grown under biotic or abiotic stresses (Thiel et al. 2004; Kazan 2006). Whether the ERF-mediated altered responses of transgenic plants to biotic and/or abiotic stresses are mediated by the EAR motif is unclear.

The aim of the present study is to gain further insight into the function of class II ERFs and the role of the EAR domain in plant response to environmental stresses and pathogen infection. Therefore, the tomato SlERF3 identified by our previous microarray analysis was isolated and analyzed for its expression under biotic and abiotic stresses. In addition, an EAR-deleted version of SlERF3, SIERF3 $\triangle R D$, was generated and characterized for its subcellular localization and transcriptional transactivation activity. Furthermore, transgenic Arabidopsis and tomato plants overexpressing SlERF3 $\triangle R D$ were generated and assessed for their response to salinity and bacterium infection. The data obtained in this study demonstrate that EAR-containing proteins may find use in crop improvement for broad-spectrum of stress tolerance through the manipulation of the EAR repressor domain.

\section{Materials and methods}

Amino acid alignment and phylogenetic tree

ERF protein sequences were obtained from the National Center for Biotechnology Information (http://www.ncbi. nlm.nih.gov), the Arabidopsis Information Resource (http://www.Arabidopsis.org/), the Sol genomic network (http://sgn.cornell.edu/index.pl), and the Rice Genome Annotation (http://rice.plantbiology.msu.edu/). Alignment of amino acid sequence was performed using the Clustal X program (Thompson et al. 1997) and further adjusted by GeneDoc software. The phylogenetic tree analysis was conducted using MEGA3.1 (Kumar et al. 2004). The phylogenetic tree was generated using the neighbor-joining method created with 1,000 bootstrap trials by use of the neighbor-joining algorithm. Percentages of bootstrap values are indicated on the tree. 
Plant materials and experimental treatment

Seeds of tomato [Solanum lycopersicon (L.) Miller cv. CL5915-93D-1-0-3] were kindly provided by the AVRDC-The World Vegetable Center (Tainan, Taiwan). Four-week-old wild-type tomato plants were raised from seeds in controlled environment chambers under a 16-h light/8-h dark cycle at $24^{\circ} \mathrm{C}$ (about $120 \mu \mathrm{mol} \mathrm{m}{ }^{-2} \mathrm{~s}^{-1}$ ), with $50 \%$ relative humidity. For chilling, salt, $R$. solanacearum, and hormone treatments, plants were grown in soil. Ethephon, an ethylene releaser, was used as ethylene replacement (Zhang and Wen 2009). Ethephon, SA, and jasmonic acid (JA) were applied on tomato leafs by spraying. For drought treatment, plants were air-dried in the growth chamber after removal from Hoagland's nutrient solution. The gene expression analyses were made using the leaf samples collected after each treatment.

RNA isolation and gene expression analysis

Total RNA isolation and northern blot analysis were performed as described previously (Hsieh et al. 2002a, b). For northern blot analysis, total RNA was separated on a $1 \%$ agarose gel and then transferred to a nylon membrane. Probes were labeled with $\left[\alpha_{-}^{32} \mathrm{P}\right] \mathrm{dCTP}$ by a random labeling method (Feinberg and Vogelstein 1983). For real-time PCR analysis, quantitative PCR was performed in triplicates with SYBR green on the ABI 7500 Real-Time PCR System (Applied Biosystems, USA) following the ABI standard protocol.

\section{Isolation and generation of SlERF3 and SlERF3 $\triangle R D$}

Partial SlERF3 cDNA was identified from subtractive cDNA libraries (Hsieh et al. 2010). Full-length SlERF3 was isolated by $5^{\prime}$ - and $3^{\prime}$-RACE with RNA specimen extracted from leaves of salt-treated wild-type plants following the manufacture's instructions (Clontech, Palo Alto, CA, USA). The re-amplified full-length SIERF3 was cloned into the pGEM-T easy vector (Promega, USA). To obtain the full-length open reading frame construct, SlERF3 was amplified using SlERF3 F1 and SIERF3 R1 primers. For SlERF3 $\triangle R D$ construct, SIERF3 $\triangle R D$ was amplified from SlERF3 full-length cDNA using SIERF3 F1 and SIERF3 $\mathrm{R} 2$ primers and cloned into the pGEM-T easy vector. All primer sequences are listed in Supplemental Table 1.

Transactivation assays

For transactivation assay, the Luc gene in pJD301 (Luehrsen et al. 1992) was replaced by SlERF3 or SIERF3 $1 R D$ as the effector plasmids. The GCC box and sequence from the RD29A gene promoter and mutant GCC box were multimerized four times and placed upstream of the minimal -42 to
+8 TATA box from the cauliflower mosaic virus (CaMV) 35S promoter. This construct was substituted for the CaMV $35 \mathrm{~S}$ promoter in pJD301, and fused to the firefly luciferase (LUC) gene as the reporter plasmid. The pBI221 plasmid containing the $\beta$-glucuronidase (GUS) gene driven by the CaMV $35 S$ promoter was used as an internal control (Hsieh et al. 2010). Transactivation assay was performed by the polyethylene glycol-mediated transformation method (Abel and Theologis 1994). Ten micrograms of reporter plasmid and $5 \mu \mathrm{g}$ of effector plasmid or control plasmid (pUC18) were co-transfected into $4 \times 10^{4}$ protoplasts with $10 \mu \mathrm{g}$ internal control plasmid pBI221. The transfected cells were incubated at $22^{\circ} \mathrm{C}$ in light for $18-20 \mathrm{~h}$, harvested by centrifugation at $100 \mathrm{~g}$ for $2 \mathrm{~min}$, and lysed in lysis buffer (Promega). Luciferase activity was measured using the Promega luciferase assay kit (E1500) on Luminometer (Berthold, Germany) according to the manufacturer's instructions, and GUS activity was determined as described (Lu et al. 1998).

Generation and molecular characterization of transgenic plants

SIERF3 and SlERF3 $A R D$ were cloned into pCAMBIA1390 driven by the CaMV35S promoter (Hsiao et al. 2007), and transgenic Arabidopsis and tomato were generated by Agrobacterium-mediated transformation as described (Hsieh et al. 2002a, b). Total RNA was isolated from leaves of $\mathrm{T}_{2}$ transgenic Arabidopsis and tomato, and untransformed plants. Transgenic Arabidopsis was confirmed by RT-PCR with specific primers SlERF3 $\triangle R D \mathrm{~F}_{1}$ and Nos-3. Primers for actin $(A c t)$ and hygromycin resistance gene (Hpt) are listed in Supplemental Table 1. The probes used for hybridization were tomato $\beta$-tubulin, hygromycin resistance gene $(\mathrm{Hpt})$, tomato pathogenesis-related protein 1 (PR1; accession number: AJ011520), PR2 ( $\beta$-1,3-glucanase, accession number: CK664757), and PR5-like (accession number: AY257487) (Schaller et al. 2000).

Stress response assays and measurement of growth characteristics of transgenic plants

Seeds of transgenic Arabidopsis were surface-sterilized as described (Brini et al. 2007) and grown under a 16-h light/8-h dark cycle at $24^{\circ} \mathrm{C}$. For germination assays, seeds were plated for 7 days on MS medium (Murashige and Skoog 1962) containing $150 \mathrm{mM} \mathrm{NaCl}$. For other analyses, 10-day-old Arabidopsis was treated with $150 \mathrm{mM} \mathrm{NaCl}$ agar medium for 7 days. The chlorophyll content, fluorescence $\left(F_{\mathrm{v}} / F_{\mathrm{m}}\right.$ ratio), and relative malondialdehyde (MDA) level were measured as described (Sanjaya et al. 2008). Transgenic and wild-type tomato were directly sown in soil for 2 weeks and soaked with $250 \mathrm{mM}$ $\mathrm{NaCl}$ solution for a few seconds at 2-day intervals for 14 days, and then chlorophyll content and fluorescence were measured. 
For bacterial wilt test, 3-week-old transgenic tomato plants whose roots were severed were inoculated with $R$. solanacearum strain Pss4 (race 1 , biovar 3$)\left(A_{600}=0.6\right.$ ) by soil-drenching (Chan et al. 2005). Wilted symptoms were observed from days 7 to 35 post-inoculation. The growth characteristics were measured for 3-month-old plants (the time includes stress treatment).

\section{Statistical analysis}

Data were analyzed by a Student's pair wise $t$ test. Statistically significant difference between treatments is indicated as follows: $* P<0.05$ and $* * P<0.01$.

\section{Results}

Isolation of SlERF3 containing the EAR domain

Recently, using microarray data from a subtractive library, it has been shown that the expression of a tomato $E R F$ mRNA was highly induced by salt and drought stress (Hsieh et al. 2010). Further characterization reveals that this gene (Unigene number SGN-U315194) encodes a protein called SIERF3. The SIERF3 protein constitutes 210 amino acids with a predicted molecular mass of $23 \mathrm{kDa}$. Amino acid sequence alignment showed that SlERF3 shares high similarity with LeERF3 (96.4\%, GenBank accession number: AY192369, isolated from S. lycopersicon cv. Microtom), LeERF2 (94.6\%, GenBank accession number: AY275554, isolated from S. lycopersicon cv. Lichun), and LeERF3b (94.6\%, GenBank accession number: AY559314, isolated from S. lycopersicon Mill cv. Alisa Craig) (Tournier et al. 2003; Zhang et al. 2005; Chen et al. 2008) (Supplemental Fig. 1). The variance in the four genes might result from different tomato cultivars or sequencing errors; alternatively, it might represent different genes with similar transcripts in tomato.

To determine the relationship between SIERF3 and other ERFs, alignment and phylogenetic analyses were carried out. Tomato ERFs identified from recent studies (Tournier et al. 2003; Wang et al. 2004; Zhang et al. 2004, 2005), class II ERFs of Arabidopsis (Fujimoto et al. 2000), rice subgroup VIIIa ERFs (Nakano et al. 2006), and EARcontaining genes from various species were analyzed. SIERF3 shares 40-59\% identity with NtEREBP5, CsERF1, AtERF11, and GmEREB4 (Fig. 1a). Phylogenetic tree analysis revealed that SIERF3 is most similar to tobacco NtEREBP5 (Fig. 1b). We should note that there are two entries of LeERF2 present in GenBank: one (GenBank accession number: AY275554) (Zhang et al. 2005) contains an EAR motif which is highly similar to SIERF3, the other (GenBank accession number: AY192368) (Tournier et al. 2003; Zhang et al. 2009; Zhang and Huang 2010) contains no EAR motif (Supplemental Fig. 1). Although the LeERF2 (AY192368) has been evidenced to modulate ethylene biosynthesis to enhance freezing tolerance (Pirrello et al. 2006; Zhang et al. 2009; Zhang and Huang 2010), the functions of SIERF3, LeERF3, LeERF3b, and LeERF2 (AY275554) remain unknown.

SlERF3 expression is induced by biotic, abiotic stresses and hormones

ERFs have been shown to play a direct regulatory role in response to multiple signal stimulation. To clarify the potential function of SlERF3 in response to different stimuli, we analyzed the temporal expression patterns of SlERF3 in tomato leaves under various biotic and abiotic stress conditions using RNA gel blot analysis. As shown in Fig. 2a, the SlERF3 transcript could barely be detected in leaves in the absence of stress conditions (designated as $0 \mathrm{~h})$. However, under chilling, drought, and salt treatments, SlERF3 transcripts accumulated substantially within $1 \mathrm{~h}$ and peaked at 2,24 , and $12 \mathrm{~h}$, respectively. In addition, SIERF3 expression was induced within $12 \mathrm{~h}$ after challenge with the bacterial pathogen $R$. solanacearum and this induction was maintained at about the same level for at least 2 days (Fig. 2b). The inductions of ethylene, JA, and SA have been shown to correlate with the onset of plant defense responses (Koornneef and Pieterse 2008). Therefore, we used quantitative RT-PCR to test the expression patterns of SlERF3 after exogenous application of ethephon, an ethylene releaser, JA, and SA. As shown in Fig. 2c, the SlERF3 transcripts were barely affected by ethephon within the first $8 \mathrm{~h}$ and increased moderately after $24 \mathrm{~h}$ of treatment. By contrast, JA treatment resulted in a rapid accumulation of SlERF3 transcripts, followed by a fast reduction of expression to a level below (4 and $8 \mathrm{~h}$ ) and equivalent to $(24 \mathrm{~h})$ SIERF3 expression in the control group. SA application led yet again to a different expression pattern: the expression of SIERF3 increased moderately and rapidly, remained constant for several hours, fell below control levels at $8 \mathrm{~h}$ of treatment, and showed a strong increase after $24 \mathrm{~h}$.

SlERF3 $\triangle R D$ acts as a GCC-mediated transcriptional activator

Sequence analysis showed that SIERF3 contains an EAR motif. To understand the function of the EAR domain within SIERF3, we generated full-length SIERF3 cDNA and EAR motif-deleted cDNA (SlERF3 $\triangle R D)$. As the nuclear localization sequence of ERF family proteins is likely located within the AP2/ERF domain (Matsuo and Banno 2008), deletion of the EAR motif from SIERF3 
Fig. 1 Amino acid alignment and phylogenetic tree of SIERF3 and ERF proteins from various species. a Comparison of the derived amino acid sequence of selected EAR motif-containing genes that have highly sequence similarity with tomato SIERF3. Dots indicate the conserved AP2/ ERF DNA-binding domain and asterisks mark the EAR motif. Amino acids identical in all proteins are shown in black; those conserved in at least three sequences are shaded.

b Phylogenic comparison of SIERF3 protein and some ERFrelated proteins. The ERF proteins used for construction of phylogenetic trees are identified from tomato (SIERF3, LeERFs, and JERFs), Arabidopsis (AtERFs), rice (OsERFs), Cucumis sativus (CsERF1), Glycine max (GmEREBP4), Gossypium hirsutum (GhERF4), Nicotiana tabacum (NtEREBP5), and Saccharum officinarum (SodERF3). Proteins containing EAR motifs are labeled as EAR a

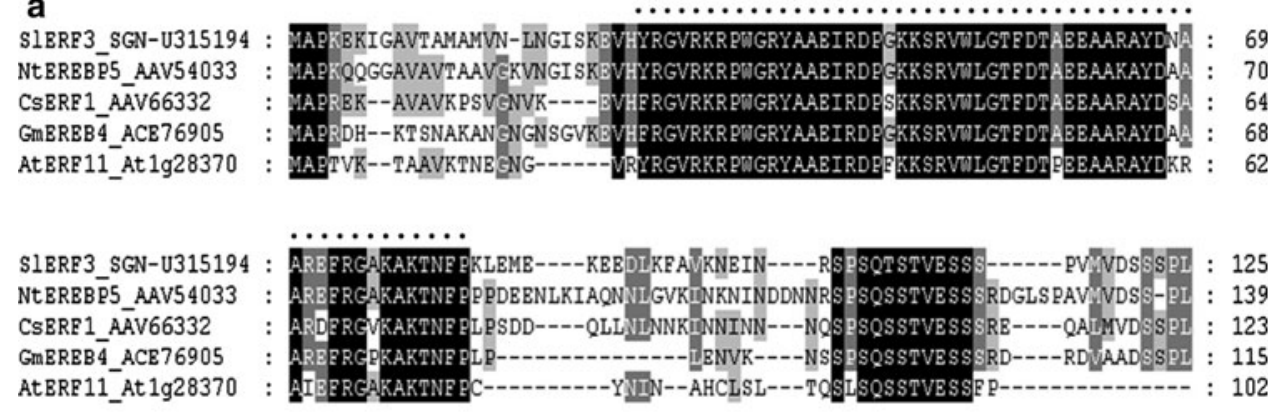

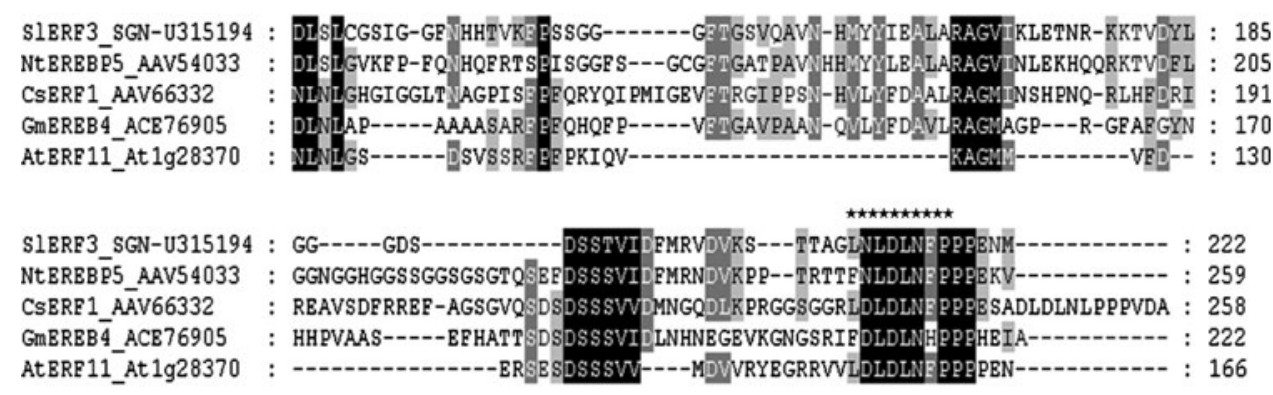

b

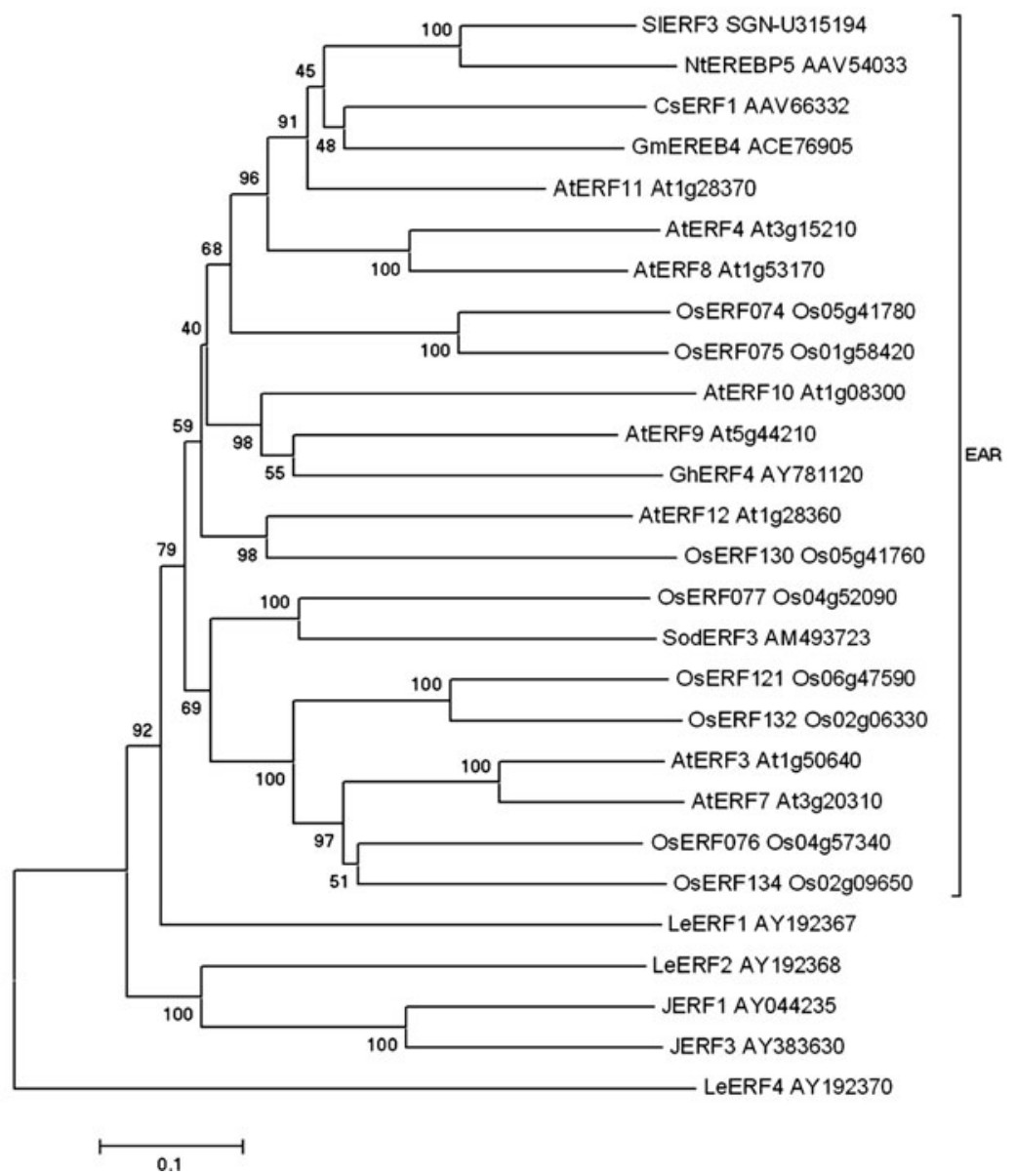

should not affect the nuclear localization of SIERF3 $\triangle R D$. Indeed, no difference in localization between SIERF3 and SIERF3 $\triangle$ RD could be detected (Supplemental Fig. 2).
The EAR domain was suggested to be responsible for the GCC-mediated transcriptional repression of AP2/ERF proteins (Ohta et al. 2001; Song et al. 2005). Therefore, 

SIERF3 under biotic and abiotic stresses, and hormone treatments, as assessed by northern blot analysis or realtime RT-PCR. a Induction of SIERF3 after chilling $\left(4^{\circ} \mathrm{C}\right)$, drought (air-drying), or salt treatments $(200 \mathrm{mM} \mathrm{NaCl})$. b Induction of SIERF3 after root-invading inoculation with the pathogen $R$. solanacearum. Ubi3 was used as an internal control in both panels. c Relative expression levels of SIERF3 after treatment with $1 \mathrm{mM}$ ethephone, $0.1 \mathrm{mM}$ methyl jasmonic acid (JA), or $1 \mathrm{mM}$ salicylic acid (SA). Relative transcripts levels were measured by real-time PCR and normalized by that of the reference gene
Fig. 2 Expression patterns of
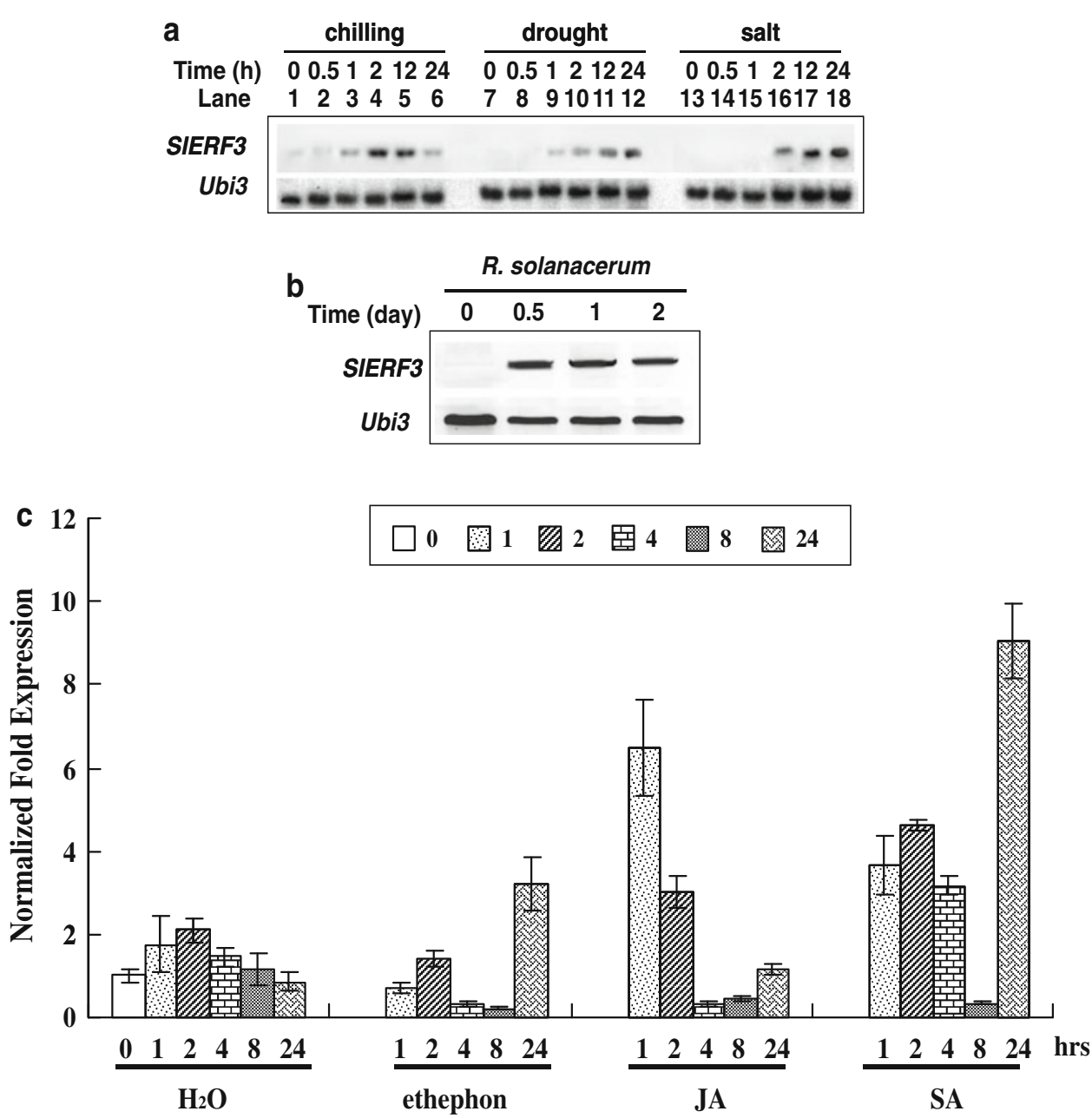

effector plasmids with SlERF3 or SlERF3 $\triangle R D$ (Fig. 3a) were used to performed transactivation assay in Arabidopsis protoplasts. A reporter gene with four tandem copies of the GCC box or a mutated GCC box (mGCC) was also used (Fig. 3b). Similar to other class II ERFs that act as transcriptional repressors, SIERF3 appeared to repress reporter gene expression since in its presence luciferase expression was reduced to $30 \%$ of the control level, whereas SIERF3 $\triangle$ RD led to a 3.8 -fold higher transactivation activity as compared with the control (Fig. 3c). By contrast, luciferase expression remained unchanged in reporter constructs $35 \mathrm{Sm}$ and $\mathrm{mGCC} 35 \mathrm{~m}$ in the absence or presence of effector. These data indicate that the EAR motif is also responsible for transcriptional activation/ repression of tomato AP2/ERF genes.

SlERF3 $\triangle R D$ transgenic tomato exhibits increased pathogenesis-related $(P R)$ gene expression and enhanced resistance to $R$. solanacearum

In order to understand how the EAR domain of SIERF3 contributes to plant stress response, we generated transgenic tomato plants with constitutive expression of SlERF3 or SIERF3 $\triangle R D$. However, SIERF3 transgenic tomato was difficult to shoot and no transgenic plant was obtained under both selection medium and normal growth condition (Supplemental Fig. 3). Therefore, further experiments of SIERF3 transgenic tomato under stresses were prohibited. On the other hand, no apparent effects on growth and development of SlERF3 $\triangle R D$ transgenic plants were observed. After antibiotic selection and genomic PCR of several SlERF3 $\triangle R D$-overexpressing tomatoes, four lines (ER3, ER8, ER10, and ER11) were selected for northern blot analysis (Fig. 4a). It has been suggested that ERF proteins may play a role in the regulation pathogenesisrelated (PR) genes containing GCC boxes, including $P R 1, P R 2, P R 3$, and $P R 5$, and thus may increase plant resistance to pathogen attack (Ohme-Takagi and Shinshi 1995; Gu et al. 2000; Park et al. 2001). To test whether the SIERF3 $\triangle R D$ protein can enhance downstream PR genes expression and pathogen resistance, homozygous progenies of SlERF3 $\triangle R D$ transgenic plants were subjected to further analyses of PR gene expression. As shown in Fig. 4a, expression of $P R 1, P R 2$, and $P R 5$ genes, which 
a Reporter plasmid

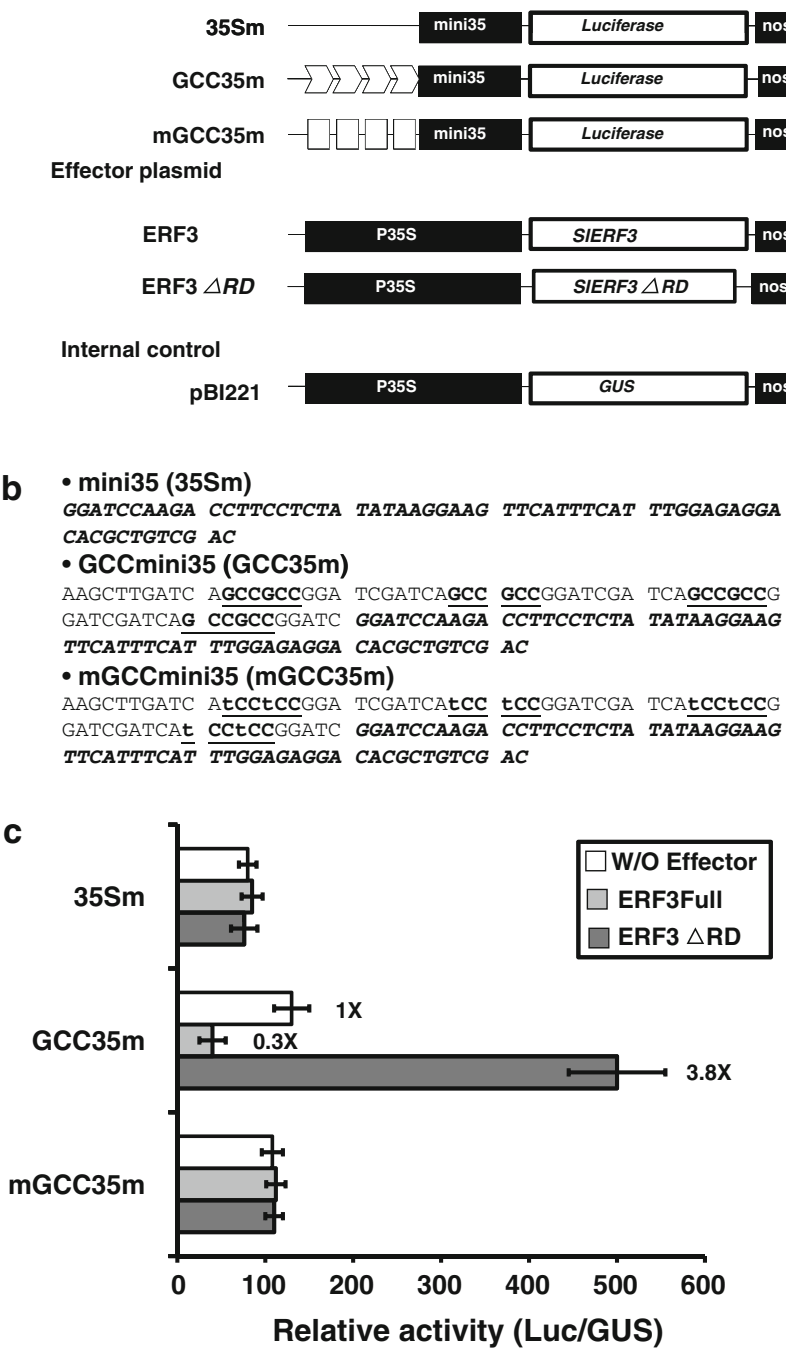

Fig. 3 Transactivation of GCC-mediated transcription by SlERF3 or SIERF3 $3 R D$ in Arabidopsis protoplasts. a Schematic diagram of reporter, effector and internal control plasmid constructs. Reporter plasmids GCC $35 \mathrm{~m}$ and mGCC $35 \mathrm{~m}$ contain four repeats of the wildtype or mutant GCC box sequence. $35 \mathrm{Sm}$ and mini35 denote unmodified and minimal CaMV35S promoter, respectively. GUS, beta-glucuronidase. b Partial sequences of reporter constructs. Sequences denoting the CaMV35S minimal promoter are shown in italics and in boldface, while GCC boxes are underlined and in boldface. $\mathbf{c}$ Relative luciferase activities in transactivation assays. The effector plasmid encoding SIERF3 or SIERF3 $\triangle R D$, and the reporter plasmid were co-transfected into protoplasts with internal control plasmid by polyethylene glycol-mediated transformation. Transcriptional activation is expressed as ratio of Luciferase (Luc) to GUS activity

contain a GCC box in their promoters, was greatly increased in SlERF3 $\triangle R D$ transgenic tomato but barely detected in wild-type plants grown under regular conditions. These data indicate that SIERF3 $\triangle \mathrm{RD}$ could act as a transcriptional activator to increase expression of defense genes.
To examine whether expression of SIERF3 $\triangle R D$ in tomato plants can enhance pathogen resistance, we performed a pathogen inoculation assay. To this end, transgenic and wild-type tomato plants were inoculated with a virulent strain of $R$. solanacearum, Pss4, by soil-drenching. As shown in Fig. 4b, $70 \%$ of the wild-type plants displayed typical wilting symptoms 14 days post-inoculation, while only $20 \%$ of the homozygous SlERF3 $\triangle R D$ transgenic plants showed a wilting phenotype. The enhancement of diseasetolerant phenotype was further confirmed by disease incidence assay. All of the wild-type plants wilted 28 days after inoculation, whereas only $30 \%$ of the transgenic plants showed symptoms 35 days post-inoculation (Fig. 4c). Consistent with these results, the photosynthetic efficiency $\left(F_{\mathrm{v}} / F_{\mathrm{m}}\right.$ ratio $)$ and chlorophyll content of SlERF3 $4 R D$ transgenic plants after bacterium infection were higher compared to wild-type plants (Fig. $4 \mathrm{~d}$, e), indicating that the level of cellular damage due to pathogen infection was much lower in the transgenic lines as compared to wild-type plants. Taken together, the results demonstrate an enhanced disease tolerance conferred by the overexpression of SIERF3 $\triangle R D$ protein in tomato plants.

Constitutive expression of SlERF3 $\triangle R D$ enhances salt tolerance in transgenic Arabidopsis

It has been reported that the expression of several GCC boxcontaining PR genes (e.g., osmotin) is normally regulated upstream in response to not only pathogen but also osmotic stress (Jia and Martin 1999). To test whether expression of SIERF3 $\triangle R D$ changes the response of a heterologous plant to salt stress, three SlERF3 $\triangle R D$ transgenic Arabidopsis lines (AER1, AER2, and AER3) were selected and analyzed. The constitutive overexpression of SIERF3ARD under normal growth conditions was confirmed by RT-PCR (Fig. 5a). Under salt stress, the SlERF3 $\triangle R D$ transgenic Arabidopsis showed normal germination and growth, while the germination and growth of the wild-type plants and the vector-only transgenic line (1301) were inhibited (Fig. 5b). In addition, the photosynthetic efficiency and chlorophyll content of SlERF3 $\triangle R D$-overexpressed lines were significantly higher than that of the control plants under high salt conditions (Fig. 5c, d). In addition, no photosynthetic defect associated with overexpression of SIERF3 $A R D$ under normal conditions was detected. Our data clearly demonstrate that overexpression of SlERF3 $\triangle R D$ enhanced salt tolerance in transgenic Arabidopsis plants.

Ectopic expression of SlERF3 $\triangle R D$ enhances salt tolerance in transgenic tomato

SIERF3 $\triangle R D$ transgenic tomatoes were further subjected to analysis for their response to salinity. While wild-type 

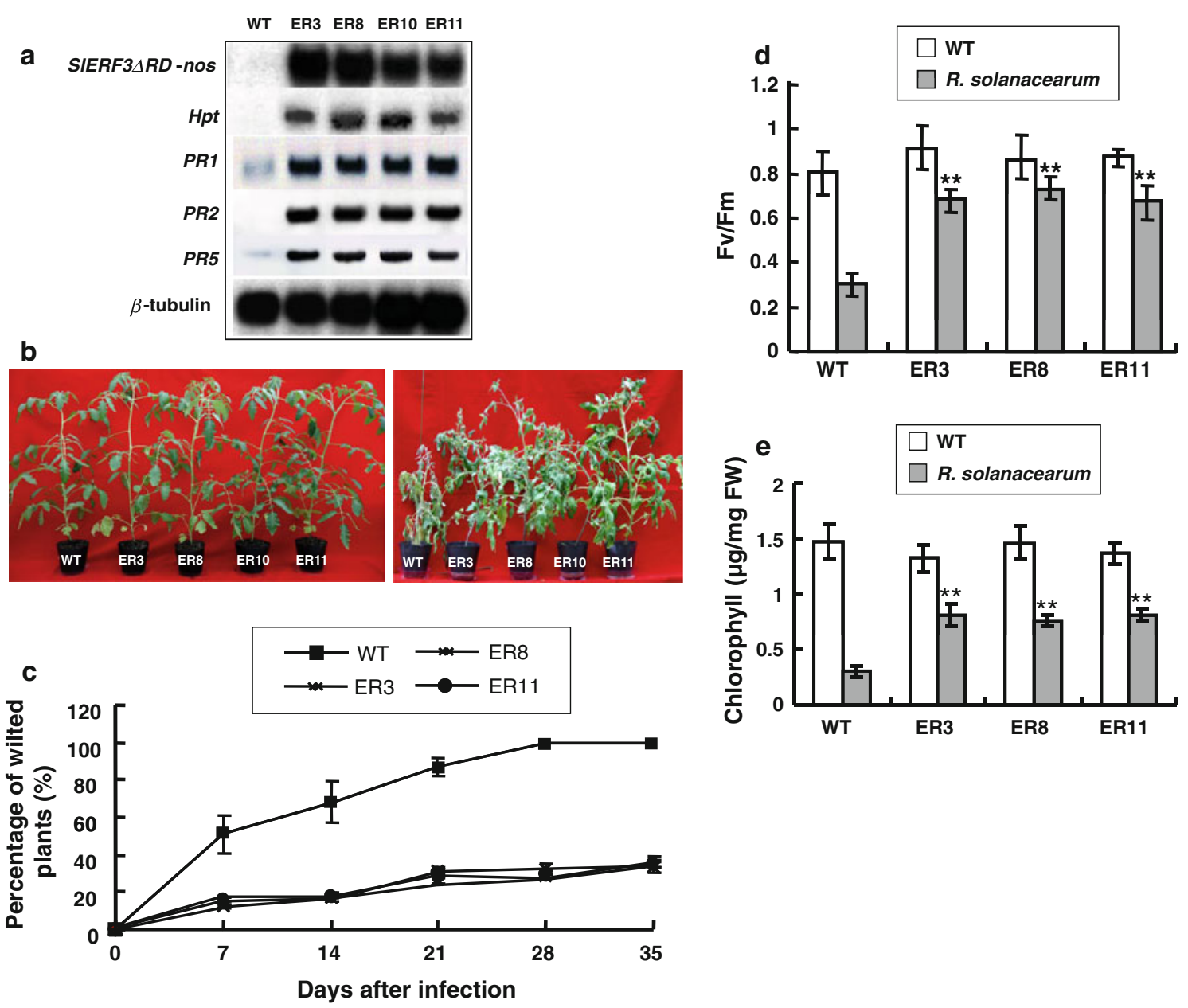

Fig. 4 Overexpression of SlERF3 $\triangle R D$ enhanced tolerance to bacterial wilt in transgenic tomato. a Northern blot analysis of SlERF3 $\triangle R D, H p t, P R 1, P R 2$, and $P R 5$ expression in wild-type plants and in SIERF3 $\triangle R D$ transgenic lines. $\beta$-tubulin was used as a loading control. b Phenotype of 3-week-old transgenic tomato and wild-type plants treated with $\mathrm{H}_{2} \mathrm{O}$ (left panel) or a virulent strain of $R$. solanacearum by root invasion for 14 days (right panel). c Percentage

plants wilted and showed necrotic and bleached leaves 14 days after treatment with $250 \mathrm{mM} \mathrm{NaCl}$, all of the transgenic plants (ER3, ER8, and ER11) remained healthy, with no signs of phenotypic damages (Fig. 6a). The photosynthetic efficiency (Fig. 6b) and chlorophyll content (Fig. 6c) of transgenic tomato were also higher than the corresponding values of wild-type plants under salinity treatment.

To further characterize these salt-tolerant transgenic plants, the level of malondialdehyde (MDA), an indicator of lipid peroxidative damage in plant tissues, was measured. We found that transgenic SlERF3 $\triangle R D$-expressing seedlings had significant lower MDA levels compared to wild-type seedlings under salt stress (Fig. 6d). The reduction in MDA levels indicates a decrease in lipid peroxidation in transgenic plants overexpressing SIERF3 $\triangle R D$. of wilted plants at different time points of infection. Wilted plants were defined as plants that showed more than $50 \%$ of leafs with wilted symptoms. Data were collected from at least 20 plants for each line. Three independent experiments were performed. d PSII photochemical efficiency $\left(F_{\mathrm{v}} / F_{\mathrm{m}}\right.$ ratio) and e Chlorophyll content of SIERF3 $\triangle R D$ transgenic lines and wild type were measured at day 7 post-inoculation $(* * P<0.01)$

These results, combined with the results described above, clearly show that the expression of SIERF3 $\triangle R D$ improves salt tolerance in transgenic tomato.

Growth characteristics of SlERF3 $\triangle R D$ transgenic tomato

The enhancement of tolerance to salt and $R$. solanacearum implied that SlERF3ARD may be a good candidate for tomato improvement. To further examine how SlERF3 $\triangle R D$ affects tomato quality, several growth characteristics of wild-type and SIERF3 $\triangle R D$ transgenic tomato under different treatments were measured. Under normal growth conditions, SlERF3 $\triangle R D$ overexpression lines showed no significant difference with wild type in fruit number, seed number, and fresh weight. However, after 
a
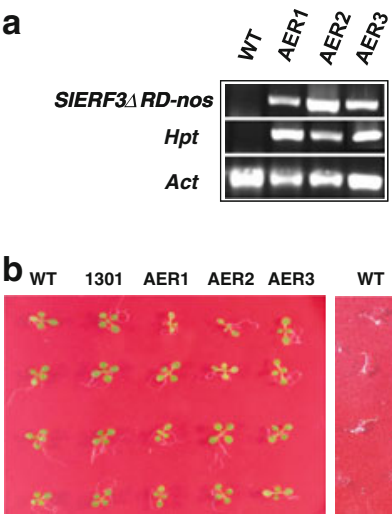

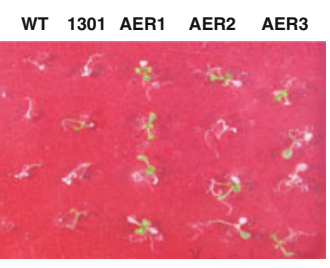

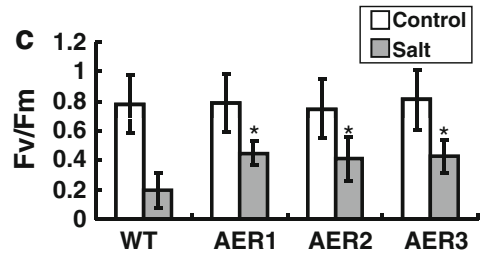

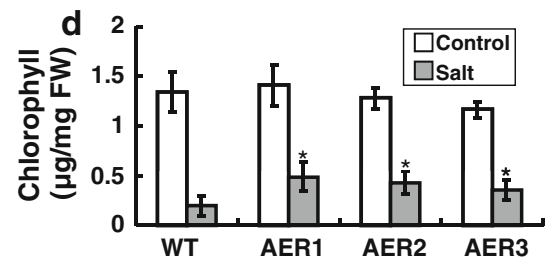

Fig. 5 SlERF3 $\triangle R D$ transgenic Arabidopsis enhanced tolerance to salt stress. a RT-PCR analysis of SlERF3 $\triangle R D$ and hygromycin resistance gene (Hpt) expression in wild-type and in transgenic lines. b Phenotype of SIERF3 $\triangle R D$ transgenic, wild type, and 1301 (vectoronly transgenic line) Arabidopsis seeds germinated for 7 days on MS medium in the absence (left panel) or presence of $150 \mathrm{mM} \mathrm{NaCl}$ (right panel). c PSII photochemical efficiency of SlERF3 $\triangle R D$ transgenic lines and wild type. d Chlorophyll content of SIERF3 $\triangle R D$ transgenic lines and wild type $(* P<0.05)$
Fig. 6 Overexpression of SIERF3 $\triangle R D$ enhanced salt tolerance in transgenic tomato. a Phenotype of 2-week-old SIERF3 $\triangle R D$ transgenic and wild-type tomato plants treated with $\mathrm{H}_{2} \mathrm{O}$ (left panel) or $250 \mathrm{mM} \mathrm{NaCl}$ (right panel) for 14 days. b PSII photochemical efficiency of SIERF3 $\triangle R D$ transgenic lines and wild type. c Chlorophyll content of SlERF3 $\triangle R D$ transgenic lines and wild type. $\mathbf{d}$ Lipid peroxidation expressed as malonaldehyde (MDA) content in SIERF3 $\triangle R D$ transgenic lines and wild type $(* P<0.05)$ a
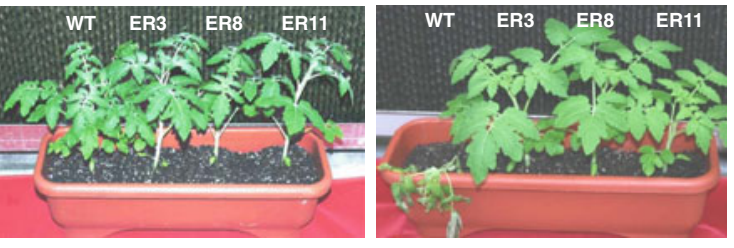

b

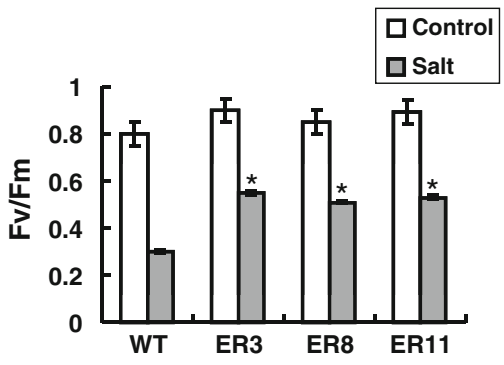

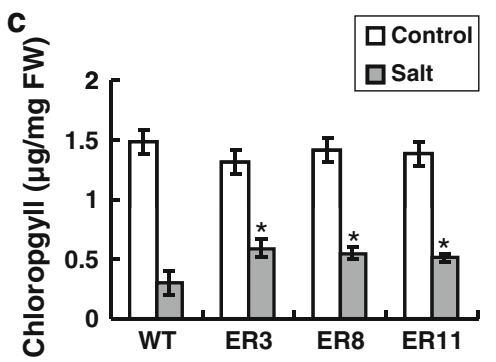

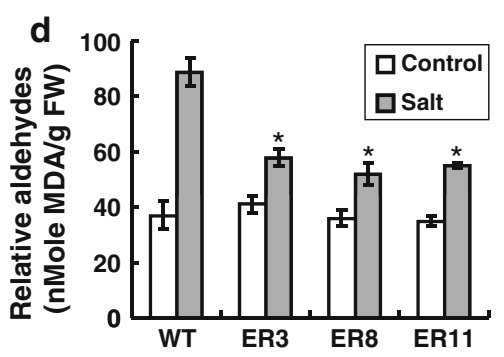

$R$. solanacearum infection, wild-type tomato produced no fruit or seeds, while the three growth characteristics of SlERF3 $\triangle R D$ transgenic lines were maintained to $60-70 \%$. Under salt stress, wild-type tomato grew few fruits and seeds. SlERF3 $\triangle R D$ transgenic lines produced much more tomato fruits and seeds compared with wild-type (Table 1). These data imply that ectopic expression of SIERF3 $\triangle R D$ not only enhances salt and $R$. solanacearum tolerance but also maintains important agronomic traits of tomato.

\section{Discussion}

Cross talk between induced ethylene, SA, and JA defensesignaling pathways is thought to contribute to induction of a powerful defense response in plants (Koornneef and Pieterse 2008). ERF genes have been proven to play key roles as regulators in three defense-signaling pathways. Two ERFs, ERF1 and ORA59, individually integrate defense signals from ethylene (ET) and jasmonate pathways and induce downstream defense-related genes including plant defensin1.2 (PDF1.2) (Lorenzo et al. 2003; Pre et al. 2008). Members of the ERF family can control defense genes positively or negatively. For example, the expression of PDF1.2, the marker gene of the ET and JA defense pathways, is induced by constitutive overexpression of ERF2 but repressed by overexpression of ERF4 in transgenic plants (Brown et al. 2003; McGrath et al. 2005). Usually, EAR-containing ERFs are involved in the repression mechanism (Ohta et al. 2001; McGrath et al. 2005). 
Table 1 Effects of various treatments on the growth characteristics in transgenic and wild-type tomato plants

\begin{tabular}{lcccc}
\hline Treatment & \multicolumn{1}{l}{ WT } & \multicolumn{1}{l}{ E3 } & \multicolumn{1}{l}{ E8 } \\
\hline Control & $20.3 \pm 2.0$ & $24.8 \pm 3.6$ & $22.4 \pm 3.2$ & $17.4 \pm 5.8$ \\
& $23.5 \pm 9.2$ & $21.2 \pm 3.0$ & $20.6 \pm 2.2$ & $20.0 \pm 1.3$ \\
& $168.4 \pm 5.1$ & $163.4 \pm 10.8$ & $168.7 \pm 9.2$ & $167.0 \pm 10.5$ \\
Ralstonia & $0 \pm 0$ & $16.3 \pm 3.1$ & $15.3 \pm 3.1$ & $13.1 \pm 2.5$ \\
& $0 \pm 0$ & $20.6 \pm 3.2$ & $15.8 \pm 6.2$ & $12.3 \pm 4.2$ \\
& $59.6 \pm 5.3$ & $111.4 \pm 3.0$ & $125.2 \pm 5.3$ & $112.3 \pm 4.2$ \\
Salt & $5.0 \pm 1.0$ & $16.3 \pm 3.1$ & $15.3 \pm 3.1$ & $13.1 \pm 2.5$ \\
& $8.0 \pm 0.5$ & $23.6 \pm 3.5$ & $22.8 \pm 4.2$ & $22.3 \pm 2.2$ \\
& $89.6 \pm 4.2$ & $132.4 \pm 4.0$ & $133.2 \pm 2.1$ & $141.5 \pm 3.2$ \\
\hline
\end{tabular}

Data in each column show, from top to bottom, fruit number (FN) per plant, seed number $(\mathrm{SN})$ per fruit, and fresh weight $(\mathrm{FW}, \mathrm{g})$ per plant. Each value represents the mean \pm standard deviation $(n=30$ individual plants). Measured plants were 3 months old, which includes the time of stress treatment

One example is AtERF7 which has been suggested to recruit a co-repressor and a histone deacetylase to block transcriptional activation (Thiel et al. 2004; Song et al. 2005). In our study, tomato SIERF3 contains an EAR motif. Our results show that overexpression of SlERF3 leads to repression of GCC-mediated transcription, suggesting that SIERF3 might act as an active repressor. Here, we showed that expression of $P R 1, P R 2$, and $P R 5$ genes were significantly induced in SlERF3 $\triangle R D$ transgenic tomato. Taken together, these results suggest that resistance to $R$. solanacearum in tomato might be achieved, at least partially, by triggering the SA-defense-signaling pathway.

In several recent studies, transgenic plants that constitutively express EAR-containing genes were generated. However, overexpression of a full-length EAR-containing gene seems to influence plant growth. Overexpression of two AP2/ERF family genes, AtERF7 and DEARl, reduced the plant size of transgenic Arabidopsis (Song et al. 2005; Tsutsui et al. 2009). Transgenic Arabidopsis constitutively expressing Zat7 or Zat10, both of which are Cys2/His2 zinc finger proteins containing an EAR motif, also showed growth suppression (Mittler et al. 2006; Ciftci-Yilmaz et al. 2007). In our study, full-length SIERF3 transgenic tomato exhibited very slow growth performance and did not develop roots; as a result, plants could not be successfully obtained. These results indicate that a precise control of the expression of EAR-containing genes is essential for the development of normal plants.

While this paper was being written, another group reported that virus-induced gene silencing of the SIERF3 gene in $R$. solanacearum-resistant tomato cultivar decreased the resistance of tomato (Chen et al. 2009). The result implied that both SIERF3 and SIERF3 $\triangle$ RD might contribute to $R$. solanacearum tolerance. Taking together, deletion of EAR motif of SIERF3 led to promoting growth of transgenic tomato without affecting $R$. solanacearum tolerance. A similar result has previously been reported with the zinc finger protein AtZat7 which also contains an EAR motif. Constitutive expression of AtZat7 resulted in growth suppression and enhanced salinity tolerance in transgenic Arabidopsis, while the deletion of the EAR motif of AtZat7 led to salt susceptibility without affecting growth suppression (Ciftci-Yilmaz et al. 2007). Collectively, these results show that EAR-containing genes may play multiple roles in response to biotic and abiotic stresses. Only partial response has been achieved by constitutively expressing genes in which the EAR motif has been deleted. Therefore, EAR motif-containing genes might act through pathways that are dependent or independent of the EAR motif.

The genetic modification of higher plants through gene engineering has become a valuable tool for the development of pathogen-resistant or stress-tolerant plants. Sweet pepper ferredoxin-like protein $(p f l p)$ gene increased the tolerance of orchid (Oncidium) to Erwinia carotovora, a plant pathogen with a wide host range (Liau et al. 2003; You et al. 2003). Overexpression of Arabidopsis tryptophan synthase beta 1 (AtTSB1) in tomato confers tolerance to cadmium stress (Sanjaya et al. 2008). In this paper, we demonstrated that a tomato gene reversed its role from a transcriptional repressor to an activator after repressordomain deletion. Overexpression of SIERF3 $\triangle R D$ enhanced tolerance to salinity and to pathogen infection in transgenic tomatoes, while agronomical traits were largely maintained. Thus, EAR motif-containing genes could be new candidates for crop improvement or plant breeding programs aimed at developing plants with superior, broadrange stress tolerance traits.

Acknowledgments We thank members of the Plant Tech Core Facility for subcellular localization assay and H. Kuhn for manuscript editing. We also appreciate the efforts of S.C. Shen and the Inverted Confocal Microscope Core Laboratory of Academia Sinica for technical assistance. This research was funded by Academia Sinica and National Science Council of the Republic of China.

\section{References}

Abel S, Theologis A (1994) Transient transformation of Arabidopsis leaf protoplasts: a versatile experimental system to study gene expression. Plant J 5:421-427

Brini F, Hanin M, Lumbreras V, Amara I, Khoudi H, Hassairi A, Pages M, Masmoudi K (2007) Overexpression of wheat dehydrin DHN-5 enhances tolerance to salt and osmotic stress in Arabidopsis thaliana. Plant Cell Rep 26:2017-2026

Brown RL, Kazan K, McGrath KC, Maclean DJ, Manners JM (2003) A role for the GCC-box in jasmonate-mediated activation of the PDF1.2 gene of Arabidopsis. Plant Physiol 132:1020-1032 
Cao Y, Song F, Goodman RM, Zheng Z (2006) Molecular characterization of four rice genes encoding ethylene-responsive transcriptional factors and their expressions in response to biotic and abiotic stress. J Plant Physiol 163:1167-1178

Chan YL, Prasad V, Sanjaya, Chen KH, Liu PC, Chan MT, Cheng CP (2005) Transgenic tomato expressing an Arabidopsis thionin (Thi2.1) driven by fruit-inactive promoter battles against phytopathogenic attack. Planta 221:386-393

Chen G, Hu Z, Grierson D (2008) Differential regulation of tomato ethylene responsive factor LeERF3b, a putative repressor, and the activator Pti4 in ripening mutants and in response to environmental stresses. J Plant Physiol 165:662-670

Chen YY, Lin YM, Chao TC, Wang JF, Liu AC, Ho FI, Cheng CP (2009) Virus-induced gene silencing reveals the involvement of ethylene-, salicylic acid- and mitogen-activated protein kinaserelated defense pathways in the resistance of tomato to bacterial wilt. Physiol Plant 136:324-335

Ciftci-Yilmaz S, Morsy MR, Song L, Coutu A, Krizek BA, Lewis MW, Warren D, Cushman J, Connolly EL, Mittler R (2007) The EAR-motif of the Cys2/His2-type zinc finger protein Zat7 plays a key role in the defense response of Arabidopsis to salinity stress. J Biol Chem 282:9260-9268

Feinberg AP, Vogelstein B (1983) A technique for radiolabeling DNA restriction endonuclease fragments to high specific activity. Anal Biochem 132:6-13

Fujimoto SY, Ohta M, Usui A, Shinshi H, Ohme-Takagi M (2000) Arabidopsis ethylene-responsive element binding factors act as transcriptional activators or repressors of GCC box-mediated gene expression. Plant Cell 12:393-404

Gu YQ, Yang C, Thara VK, Zhou J, Martin GB (2000) Pti4 is induced by ethylene and salicylic acid, and its product is phosphorylated by the Pto kinase. Plant Cell 12:771-786

Gu YQ, Wildermuth MC, Chakravarthy S, Loh YT, Yang C, He X, Han Y, Martin GB (2002) Tomato transcription factors pti4, pti5, and pti6 activate defense responses when expressed in Arabidopsis. Plant Cell 14:817-831

Hiratsu K, Matsui K, Koyama T, Ohme-Takagi M (2003) Dominant repression of target genes by chimeric repressors that include the EAR motif, a repression domain, in Arabidopsis. Plant $\mathrm{J}$ 34:733-739

Hsiao P, Sanjaya, Su RC, da Silva JAT, Chan MT (2007) Plant native tryptophan synthase beta 1 gene act as a non-antibiotic selection marker for plant transformation. Planta 225:897-906

Hsieh TH, Lee JT, Charng YY, Chan MT (2002a) Tomato plants ectopically expressing Arabidopsis CBF1 show enhanced resistance to water deficit stress. Plant Physiol 130:618-626

Hsieh TH, Lee JT, Yang PT, Chiu LH, Charng YY, Wang YC, Chan MT (2002b) Heterology expression of the Arabidopsis C-repeat/ dehydration response element binding factor 1 gene confers elevated tolerance to chilling and oxidative stresses in transgenic tomato. Plant Physiol 129:1086-1094

Hsieh TH, Li CW, Su RC, Cheng CP, Sanjaya, Tsai YC, Chan MT (2010) A tomato bZIP transcription factor, SlAREB, is involved in water deficit and salt stress response. Planta 231:1459-1473

Jia Y, Martin GB (1999) Rapid transcript accumulation of pathogenesis-related genes during an incompatible interaction in bacterial speck disease-resistant tomato plants. Plant Mol Biol 40:455-465

Jin LG, Liu JY (2008) Molecular cloning, expression profile and promoter analysis of a novel ethylene responsive transcription factor gene GhERF4 from cotton (Gossypium hirstum). Plant Physiol Biochem 46:46-53

Jofuku KD, den Boer BG, Van Montagu M, Okamuro JK (1994) Control of Arabidopsis flower and seed development by the homeotic gene APETALA2. Plant Cell 6:1211-1225
Kazan K (2006) Negative regulation of defense and stress genes by EAR-motif-containing repressors. Trends Plant Sci 11:109-112

Koornneef A, Pieterse CM (2008) Cross talk in defense signaling. Plant Physiol 146:839-844

Kumar S, Tamura K, Nei M (2004) MEGA3: integrated software for molecular evolutionary genetics analysis and sequence alignment. Brief Bioinform 5:150-163

Liau CH, You SJ, Prasad V, Hsiao HH, Lu JC, Yang NS, Chan MT (2003) Agrobacterium-mediated genetic transformation of an Oncidium orchid. Plant Cell Rep 21:993-998

Lorenzo O, Piqueras R, Sanchez-Serrano JJ, Solano R (2003) ETHYLENE RESPONSE FACTOR1 integrates signals from ethylene and jasmonate pathways in plant defense. Plant Cell 15:165-178

Lu CA, Lim EK, Yu SM (1998) Sugar response sequence in the promoter of a rice alpha-amylase gene serves as a transcriptional enhancer. J Biol Chem 273:10120-10131

Luehrsen KR, DeWet JR, Walbot V (1992) Transient expression analysis in plants using the firefly luciferase reporter gene. Meth Enzymol 216:397-414

Matsui K, Ohme-Takagi M (2009) Detection of protein-protein interactions in plants using the transrepressive activity of the EAR motif repression domain. Plant J 61:570-578

Matsuo N, Banno H (2008) The Arabidopsis transcription factor ESR1 induces in vitro shoot regeneration through transcriptional activation. Plant Physiol Biochem 46:1045-1050

McGrath KC, Dombrecht B, Manners JM, Schenk PM, Edgar CI, Maclean DJ, Scheible WR, Udvardi MK, Kazan K (2005) Repressor- and activator-type ethylene response factors functioning in jasmonate signaling and disease resistance identified via a genome-wide screen of Arabidopsis transcription factor gene expression. Plant Physiol 139:949-959

Mittler R, Kim Y, Song L, Coutu J, Coutu A, Ciftci-Yilmaz S, Lee H, Stevenson B, Zhu JK (2006) Gain- and loss-of-function mutations in Zat10 enhance the tolerance of plants to abiotic stress. FEBS Lett 580:6537-6542

Murashige T, Skoog F (1962) A revised medium for rapid growth and bioassay with tobacco tissue cultures. Physiol Plant 15:473-497

Nakano T, Suzuki K, Fujimura T, Shinshi H (2006) Genome-wide analysis of the ERF gene family in Arabidopsis and rice. Plant Physiol 140:411-432

Narusaka Y, Nakashima K, Shinwari ZK, Sakuma Y, Furihata T, Abe H, Narusaka M, Shinozaki K, Yamaguchi-Shinozaki K (2003) Interaction between two cis-acting elements, ABRE and DRE, in ABA-dependent expression of Arabidopsis rd29A gene in response to dehydration and high-salinity stresses. Plant $\mathbf{J}$ 34:137-148

Ohme-Takagi M, Shinshi H (1995) Ethylene-inducible DNA binding proteins that interact with an ethylene-responsive element. Plant Cell 7:173-182

Ohta M, Matsui K, Hiratsu K, Shinshi H, Ohme-Takagi M (2001) Repression domains of class II ERF transcriptional repressors share an essential motif for active repression. Plant Cell 13:1959-1968

Onate-Sanchez L, Anderson JP, Young J, Singh KB (2007) AtERF14, a member of the ERF family of transcription factors, plays a nonredundant role in plant defense. Plant Physiol 143:400-409

Park JM, Park CJ, Lee SB, Ham BK, Shin R, Paek KH (2001) Overexpression of the tobacco Tsil gene encoding an EREBP/ AP2-type transcription factor enhances resistance against pathogen attack and osmotic stress in tobacco. Plant Cell 13:1035-1046

Pirrello J, Jaimes-Miranda F, Sanchez-Ballesta MT, Tournier B, Khalil-Ahmad Q, Regad F, Latche A, Pech JC, Bouzayen M (2006) S1-ERF2, a tomato ethylene response factor involved in 
ethylene response and seed germination. Plant Cell Physiol 47:1195-1205

Pre M, Atallah M, Champion A, De Vos M, Pieterse CM, Memelink J (2008) The AP2/ERF domain transcription factor ORA59 integrates jasmonic acid and ethylene signals in plant defense. Plant Physiol 147:1347-1357

Qin F, Sakuma Y, Tran LS, Maruyama K, Kidokoro S, Fujita Y, Fujita M, Umezawa T, Sawano Y, Miyazono K, Tanokura M, Shinozaki K, Yamaguchi-Shinozaki K (2008) Arabidopsis DREB2A-interacting proteins function as RING E3 ligases and negatively regulate plant drought stress-responsive gene expression. Plant Cell 20:1693-1707

Sakuma Y, Liu Q, Dubouzet JG, Abe H, Shinozaki K, YamaguchiShinozaki K (2002) DNA-binding specificity of the ERF/AP2 domain of Arabidopsis DREBs, transcription factors involved in dehydration- and cold-inducible gene expression. Biochem Biophys Res Commun 290:998-1009

Sanjaya, Hsiao PY, Su RC, Su RC, Ko SS, Tong CG, Yang RY, Chan MT (2008) Overexpression of Arabidopsis thaliana tryptophan synthase beta 1 (AtTSB1) in Arabidopsis and tomato confers tolerance to cadmium stress. Plant Cell Environ 31:1074-1085

Sasaki K, Mitsuhara I, Seo S, Ito H, Matsui H, Ohashi Y (2007) Two novel AP2/ERF domain proteins interact with cis-element VWRE for wound-induced expression of the Tobacco tpoxN1 gene. Plant J 50:1079-1092

Schaller A, Roy P, Amrhein N (2000) Salicylic acid-independent induction of pathogenesis-related gene expression by fusicoccin. Planta 210:599-606

Song CP, Agarwal M, Ohta M, Guo Y, Halfter U, Wang P, Zhu JK (2005) Role of an Arabidopsis AP2/EREBP-type transcriptional repressor in abscisic acid and drought stress responses. Plant Cell 17:2384-2396

Thiel G, Lietz M, Hohl M (2004) How mammalian transcriptional repressors work. Eur J Biochem 271:2855-2862

Thompson JD, Gibson TJ, Plewniak F, Jeanmougin F, Higgins DG (1997) The CLUSTAL_X windows interface: flexible strategies for multiple sequence alignment aided by quality analysis tools. Nucleic Acids Res 25:4876-4882

Tournier B, Sanchez-Ballesta MT, Jones B, Pesquet E, Regad F, Latche A, Pech JC, Bouzayen M (2003) New members of the tomato ERF family show specific expression pattern and diverse DNA-binding capacity to the GCC box element. FEBS Lett 550:149-154

Trujillo LE, Sotolongo M, Menendez C, Ochogavia ME, Coll Y, Hernandez I, Borras-Hidalgo O, Thomma BP, Vera P, Hernandez
L (2008) SodERF3, a novel sugarcane ethylene responsive factor (ERF), enhances salt and drought tolerance when overexpressed in tobacco plants. Plant Cell Physiol 49:512-525

Tsutsui T, Kato W, Asada Y, Sako K, Sato T, Sonoda Y, Kidokoro S, Yamaguchi-Shinozaki K, Tamaoki M, Arakawa K, Ichikawa T, Nakazawa M, Seki M, Shinozaki K, Matsui M, Ikeda A, Yamaguchi J (2009) DEAR1, a transcriptional repressor of DREB protein that mediates plant defense and freezing stress responses in Arabidopsis. J Plant Res 122:633-643

van der Fits L, Memelink J (2001) The jasmonate-inducible AP2/ ERF-domain transcription factor ORCA3 activates gene expression via interaction with a jasmonate-responsive promoter element. Plant J 25:43-53

Wang H, Huang Z, Chen Q, Zhang Z, Zhang H, Wu Y, Huang D, Huang $R$ (2004) Ectopic overexpression of tomato JERF3 in tomato actives downstream gene expression and enhances salt tolerance. Plant Mol Biol 55:183-192

Yang Z, Tian L, Latoszek-Green M, Brown D, Wu K (2005) Arabidopsis ERF4 is a transcriptional repressor capable of modulating ethylene and abscisic acid responses. Plant Mol Biol 58:585-596

You SJ, Liau CH, Huang HE, Feng TY, Prasad VP, Hsiao HH, Lu JC, Chan MT (2003) Sweet pepper ferredoxin-like protein ( $p f p$ ) gene as a novel selection marker for orchid transformation. Planta 217:60-65

Zhang Z, Huang R (2010) Enhanced tolerance to freezing in tobacco and tomato overexpressing transcription factor TERF2/LeERF2 is modulated by ethylene biosynthesis. Plant Mol Biol 73:241-249

Zhang W, Wen CK (2009) Preparation of ethylene gas and comparison of ethylene responses induced by ethylene, ACC, and ethephon. Plant Physiol Biochem 48:45-53

Zhang H, Huang Z, Xie B, Chen Q, Tian X, Zhang X, Zhang H, Lu X, Huang D, Huang R (2004) The ethylene-, jasmonate-, abscisic acid- and NaCl-responsive tomato transcription factor JERF1 modulates expression of GCC box-containing genes and salt tolerance in tobacco. Planta 220:262-270

Zhang H, Zhu B, Yu B, Hao Y, Fu D, Xu W, Luo Y (2005) Cloning and DNA-binding properties of ethylene response factor, LeERF1 and LeERF2, in tomato. Biotechnol Lett 27:423-428

Zhang Z, Zhang H, Quan R, Wang XC, Huang R (2009) Transcriptional regulation of the ethylene response factor LeERF2 in the expression of ethylene biosynthesis genes controls ethylene production in tomato and tobacco. Plant Physiol 150:365-377 